\title{
La sociedad del conocimiento: inclusión o exclusión
}

\section{The Knowledge Society: Inclusion or Exclusion}

\author{
Herminia Moreno Ríos \\ Escuela Secundaria Profesor Jesús M. Campos \\ Escuela Primaria Lic. Benito Juárez. Cadereyta Jiménez \\ Nuevo León, Méxic \\ hermy_11@hotmail.com
}

Rosa Amelia Velázquez Martínez Escuela Secundaria Nocturna \# 4 "Sra. Virginia Treviño de Collins", Santa Catarina N.L. Universidad Autónoma de Nuevo León, Preparatoria Técnica Industrial "Pablo Livas" Monterrey, Nuevo León, Méxic

vemar_1101@hotmail.com

Recibido: 10-IX-2011 • Aceptado: 11-III-2012 • Corregido: 30-XI-2012

\begin{abstract}
Resumen: En la historia de todas las sociedades, el conocimiento ha sido monopolizado por una élite, que lo utiliza para conservar el poder, se crearon limitantes para excluir a la masa de los saberes relevantes que otorgan la supremacía a unos y confinan a la mayoría a un trabajo físico o menos remunerado. En la actualidad surge el término Sociedad del Conocimiento para designar a nuestra sociedad; denominación que pareciera poner al alcance de todos tan preciado saber; con este trabajo pretendemos analizar si este beneficio es para todos; o es una forma disfrazada para continuar excluyendo y marginando a una gran parte de la sociedad; ya que la realidad muestra solo a una humanidad que tiene mayor acceso a la información; por la tecnología de la comunicación y el Internet; pero esa información aún debe transformarse en conocimiento utilitario, lo que en realidad existe es una sociedad de la información, que realmente no asegura que se pueda compartir con todos, los beneficios que brinda el conocimiento conductor de las sociedades; únicamente ofrece los saberes necesarios para competir en un mundo globalizado en donde cada individuo, según sus capacidades y competencias, debe encontrar su lugar.
\end{abstract}

Palabras clave: Sociedad del conocimiento, Sociedad

\section{Introducción}

En este trabajo pretendemos realizar una mirada a través de la historia, valorando en diversas culturas, como el descubrimiento de distintos saberes, en diferentes momentos de la vida del hombre; han permitido el avance de la humanidad y como este conocimiento fue resguardado y monopolizado por una élite, que gracias a este saber y el uso de la fuerza; mantuvo su estadía en el poder. Estos conocimientos fueron trasmitidos únicamente a sus descendientes; con el fin de asegurar su supremacía sobre los demás, excluyendo con esto al resto del pueblo, a quien se le confirió a otras labores utilitarias y necesarias para la sociedad pero menos remunerativas e importantes. El propósito de está reflexión es analizar si el término 
de la información, conocimiento, exclusión.

\begin{abstract}
In the history of every society, knowledge has been monopolized by an elite, who uses it to keep power. Limitations were set to exclude the mass from relevant knowledge. The supremacy was granted to only some and the majority were confined to physical or less paid jobs. Recently, the term knowledge society was coined to designate our current society, which seems to put at everybody's hand such precious knowledge. With this research, we intend to analyze whether this benefit is for everyone or if it is a disguised form to continue excluding and marginalizing a large part of the society, since the reality shows that humankind has greater access to only information through the technologies of information and communication and the Internet, but, that information needs yet to become utilitarian knowledge. What we actually find is an information society that does not really ensure that it can be shared with everybody. The benefits provided by the driving knowledge of societies only provide the necessary knowledge to compete in a globalized world in which each individual, according to their abilities and skills, must find its place.
\end{abstract}

Keywords: Knowledge society, information society, knowledge exclusion.
Sociedad del Conocimiento que surge para designar a nuestra sociedad actual; puede ofrecer un futuro prometedor que logre romper con lo sucedido en el pasado; o es una forma más de exclusión. Para ello es necesario conocer ¿Qué consideramos como Sociedad del Conocimiento y Sociedad de la Información? Pues se han manejado como sinónimos, es importante analizar dichas designaciones; para comprender mejor esta sociedad; para esto expondremos las opiniones de diversos autores a fin de analizar las opiniones que tienen cada uno de ellos hacia este concepto; y ¿Si el conocimiento que propone es en verdad tal?, o si es una máscara más para disfrazar la exclusión de la masa como ha sucedido en el transcurso de la historia.

\section{Historia de las sociedades y su relación con el conocimiento}

Desde los orígenes bíblicos el conocimiento ha sido fuente de poder, así tentó la serpiente a Eva ofreciéndole que al comer del fruto prohibido; podría obtener la ciencia y el conocimiento que los haría como Dioses. Ese fue el inició; de una ardua búsqueda del bienestar perdido en el Edén.

Según la mitología griega, el conocimiento también era un tesoro sólo poseído por los Dioses; Prometeo tuvo que robar el fuego para llevarles un poco de calor a los indefensos hombres; compartiendo con ellos este, y otros conocimientos de relevante importancia para la humanidad y por esta y otras osadías también fue castigado (Garibay, 1989).

El descubrimiento del fuego cambió ciertamente la vida del hombre; le dio luz, lo protegió del frío y de los animales, le sirvió para fundir metales y cocinar los alimentos así transformó sus hábitos de consumir la carne cruda; lo que le permitió tener una mejor digestión, dormir mejor y tener sueños lúcidos; que después trató de convertir en realidad; soñar le permitió crear; y la creación era sólo atributo de los Dioses (Mautino, 2009). 
Después el hombre descubrió la forma de producir los alimentos, con este conocimiento se inició la primera gran revolución: la agrícola que cambió la forma de vivir del ser humano, pues lo hizo sedentario, con el excedente de alimento asegurado, el hombre tuvo tiempo de dedicar sus energías en otras actividades; así aparecieron las primeras aldeas y después las grandes civilizaciones; donde el conocimiento fraccionado y dominado por cada ser los llevó a la especialización del trabajo, así aparecieron las labores artesanales y con este "saber hacer" se dio inicio a la segunda revolución: la artesanal.

En las civilizaciones agrícolas, el conocimiento para que el ciclo de la producción de la tierra se diera exitosamente, fue adquirido con base en la observación, por hombres que dedicaron su tiempo a develar los secretos que guardaban las estrellas, a través de la creación del calendario; los Egipcios tenían dos: uno era de dominio público y otro era solo conocido por los sacerdotes pero este descubrimiento lo usaron para poseer la supremacía sobre los demás, pues al saber medir el tiempo e identificar los cambios en las estaciones del año, aprendieron con ello los tiempos de siembra y cosecha esenciales en una comunidad agrícola (Contreras, s.f.) este fue uno de los conocimientos que les dio poder y les permitió que fueran considerados elegidos de los Dioses para gobernar, obteniendo así la facultad para dirigir a la masa; esto está plasmado en la historia a través de diversas culturas variando solo el nombre de los distintos estilos de gobierno. El sacerdocio, la religión o forma de pensar y creer, hizo alianza con la forma de gobierno y en algunos casos se unió en uno solo para guiar y dominar una civilización.

Esto permitió la existencia de las grandes civilizaciones esclavistas: como la egipcia, griega, mesopotámica etc., sociedades en las que el conocimiento estaba resguardado en manos de unos pocos. Los egipcios tenían escuelas muy herméticas llamadas Misterios a la que acudían gentes de las clases superiores (Leadbeater, 2009) los griegos tuvieron también grandes maestros y filósofos que enseñaban a los jóvenes nobles (Martín, 1999). En Mesopotamia se han encontrado tablillas en las que se resguardaba el conocimiento y era como en el resto de las demás culturas, solo del dominio de una élite que gobernaba y enseñaba únicamente a sus descendientes los saberes que les permitirían continuar dirigiendo a un pueblo ignorante y esclavizado que fue fanatizado y convencido de trabajar para agradar a sus dioses, así como adquirir los recursos para sobrevivir, convivir en paz y armonía dentro de esa sociedad.

No solo las civilizaciones europeas utilizaron este recurso, en América también sus culturas elitizaron el conocimiento. Los Aztecas por ejemplo tenían dos escuelas; "el Telpuchcallí o casa de los jóvenes para la educación corriente, y el Calmécac, para el adiestramiento de los deberes sacerdotales" (Vaillant, 1985) los nobles hijos de los gobernantes y sacerdotes acudían al Calmécac donde les impartían enseñanza especial en deberes sacerdotales y de mando. Al Telpochcalli acudían los macehuales que eran la gente del pueblo a los que "su clan les enseñaba civismo, historia y tradiciones, la obediencia a las normas religiosas el empleo de las armas, las artes y los oficios" (Vaillant, 1985) así como sembrar, arreglar canales y otras labores encaminadas a la siembra.

Tanto en Europa como en América el cristianismo contribuyó al derrumbe de las sociedades esclavistas, al predicar la igualdad entre los hombres, pero también llevó a la humanidad a su etapa más oscurantista y alejada del conocimiento, mediante la Santa Inquisición, condenaron todo conocimiento alejado del dogma religioso, como una herejía y con el saber enclaustrado en una abadía (Martín, 1999) la iglesia mantuvo su dominio durante la Edad Media, enseñando solo lo que le permitía sostener su ideología.

El conocimiento de la ciencia aplicado a la tecnología contribuyó a inclinar la 
balanza a quienes lo poseían, como la supremacía en armamentos permitió la conquista de América con lo cual se destruyeron los antiguos regímenes opresivos para dar paso a un nuevo dominio: el de la espada y la cruz en el nuevo mundo descubierto.

Aunque a veces estos saberes concretados en inventos han desestabilizado instituciones importantes como la creación de la imprenta occidental a mediados del siglo XV (Enciclopedia Hispánica, 1993) que permitió la difusión de la Biblia, considerado uno de los primeros libros que se publicaron y con el cual se difundió la palabra de Dios, que al fin pudo ser leída por quienes poseían esa habilidad entonces la cuestionaron, así el cristianismo se fragmentó en diversas religiones que tenían un mismo origen pero se practicaba con preceptos diferentes, según su interpretación bíblica. Con el invento de la imprenta empezó a haber mayor difusión de las ideas, pero la sociedad aún no podía desprenderse del dogma.

Hasta que llegó el siglo XVIII, el siglo de las luces, con el movimiento intelectual conocido como la Ilustración, que dio paso a la razón como medio para conocer, permitiendo acercarse más a la verdad, en una lucha contra el dogmatismo. Durante el Renacimiento el conocimiento se difundió en la enciclopedia, que reunía todo el saber acumulado hasta esa época (Enciclopedia Hispánica, 1993), al mismo tiempo se dieron a conocer nuevos descubrimientos; puesto que hasta entonces se pudieron difundir sin la limitante inquisidora de la iglesia. Fue un verdadero renacimiento de las artes y la ciencia, donde el conocimiento convertido en inventos y descubrimientos salía a la luz en amplia difusión y estaba disponible en las universidades como en los libros; pero la forma de llegar a él seguía siendo sólo privilegio de los que tuvieran la habilidad de saber leer.

A mediados del siglo XVIII se da también una nueva revolución: La Revolución Industrial y con ello se cambia el paradigma de hacer las cosas de manera artesanal a ser elaborado por las máquinas, proliferan las que funcionan con vapor y carbón pero posteriormente son sustituidas por la nueva energía que mueve al mundo que son los hidrocarburos.

Esta nueva forma de transformar la materia prima por medio de la industria sitúa, con el tiempo, a los países poseedores del conocimiento de saber diseñar esta tecnología; en los países más ricos del mundo, otra vez es el conocimiento él que otorga el poder económico; a finales del siglo XIX permite ejercer una dominación sobre los países pobres mediante el imperialismo; (Braillard \& Senarclens, 1982) al obtener de ellos la materia prima y después devolverla transformada en productos utilitarios, que eran vendidos a un precio más elevado, obteniendo con ello grandes ganancias pero nunca ponían a su alcance ni la técnica ni la ciencia para que ellos elaboraran su propia industria; ese sigue siendo el conocimiento resguardado que les otorga la supremacía y para asegurarla actualizan, renuevan y mejoran en forma permanente su maquinaria, creando así una gran brecha tecnológica inalcanzable entre los productores de tecnología con los importadores de esta y creando otras formas de dominio con el neo-colonialismo (Vargas,2004).

Con la Revolución Industrial y el auge del sistema capitalista, cambiaron otra vez las relaciones humanas, retrocediendo en la historia; al finalizar las sociedades esclavistas; el esclavo se convirtió en siervo, los más afortunados que aprendieron un oficio y se dedicaron a fabricar algo se convirtieron en artesanos, que empleando herramientas simples producían objetos en pequeñas cantidades que luego vendían, a finales de la Edad Media "los artesanos trabajaban para un contratista quien les encargaba la construcción de los productos que necesitaba para aumentar la producción repartieron las tareas entre varios artesanos de acuerdo a sus habilidades, así este, se convirtió en un trabajador asalariado dependiente de quien disponía 
el capital” (Mautino, 2009,p.44 ). Así se aprecia el cambio de esclavo a siervo y de siervo a obrero de los hombres que poseían como único conocimiento el saber utilizar su fuerza laboral y artesanal, con la cual intercambiaba la aportación de su trabajo a cambio de bajos salarios. El que tenía el capital era el dueño de las fábricas y de la producción, dueño de la tecnología y del conocimiento, de los mecanismos para producir y enriquecerse, el capital le daba el acceso a la adquisición de la tecnología de vanguardia.

A través de la historia vemos como el ser humano se ha ido apropiando de cierto tipo de conocimientos, con los que ha mejorado en forma paulatina sus condiciones de vida. Las sociedades han vivido grandes transformaciones, el hombre se ha adaptado a diversas formas de gobierno y de dominación, se ha avanzando en las conquistas de derechos y saberes, pero el conocimiento que permite tener la visión de conducir al mundo según las necesidades actuales no se ha compartido, como lo denuncia la filosofía marxista (Martín, 1999), aún más, se ha creado con él, nuevas necesidades a las masas, con la finalidad de que los innovadores de la tecnología puedan seguir enriqueciéndose; esto sitúa a la vanguardia a unos y excluye a la gran mayoría.

"En el transcurso de la historia de la educación, la tecnología por su vinculación con las actividades artesanales de carácter práctico, fue considerada por las élites culturales como una actividad inferior" (Matuino,2009); quizás por ello la educación tecnológica se ha extendido en el mundo a partir de la reforma de los años setenta para responder a demandas de las necesidades sociales cuyo propósito principal es preparar a los y las jóvenes para el mundo del trabajo así se puede apreciar que el conocimiento que se ha proporcionado a la población ha sido solo los saberes necesarios e indispensables, únicamente lo que conviene o en lo se requiere capacitarse para la producción, para poder hacer uso de las máquinas más no para crear la maquinaria y poder terminar con la dependencia económica; ese es un conocimiento se ha sido resguardado con una amplia serie de estrategias por los países industrializados para su único beneficio (Braillard \& Senarclens, 1982).

\section{La tecnología un satisfactor que transformó a la sociedad}

A mediados de los años setentas irrumpe en el mundo una tecnología que había sido creada para el ejército estadounidense y posteriormente se extendió con el uso de las computadoras y las redes de comunicación y el Internet, al dominio público; viene a reformar la forma de tener acceso a la información, creándose así la revolución de las nuevas tecnologías.

En la actualidad el mundo entra en un proceso de globalización donde se pretende aprovechar los talentos de toda la humanidad para producir y crear la sociedad del futuro, surge así el término: Sociedades del Conocimiento para llamar de alguna manera a la sociedad de esta época, esta terminología fue utilizada por primera vez en 1969 por Peter Drucker, este visionario considera al conocimiento como el centro de la producción de la riqueza.

Pero como puede ser esto posible, en distintas épocas de la historia de la humanidad se ha visto como ha variado el factor que produce la riqueza; en el feudalismo fue la tierra, en el capitalismo el trabajo y al iniciar el siglo XXI el conocimiento es la fuerza motriz de la economía, el conocimiento se convierte en la fuerza de transformación de la producción "La clave de este proceso es que el conocimiento introduce un espacio de innovación permanente e integral que articula el conocimiento autosostenido, incorporando un desarrollo más equitativo" (Montes, 2001). La compiladora de los Cuadernos de Iberoamérica, Rosa Isabel Montes Mendoza y su equipo de colaboradores consideran que la innovación juega 
un papel importante en el crecimiento económico así como la mano de obra de calidad capacitada; ya no es la máquina que produce en serie ahora se requiere también al capital humano capaz de investigar, desarrollar e innovar para crear y producir y para ello es necesario vincular al sector productivo con la educación en general; desde el desarrollo educativo deben iniciarse las reformas requeridas para lograr el perfil del ciudadano que demanda esta sociedad.

Esto es un propósito también de la Organización de las Naciones Unidas para la Educación, la Ciencia y la Cultura (UNESCO), para quien la Sociedad del Conocimiento se preocupa por el desarrollo del individuo y busca crear en él "la capacidad de identificar, producir, tratar, transformar, difundir y utilizar los conocimientos necesarios para el desarrollo humano", basado en sus derechos. Este organismo considera que esta sociedad "abre el camino a la humanización del proceso de mundialización" (UNESCO, 2005, p. 29).

Por ello pretende velar para que antes del año 2015 todos los niños y niñas tengan enseñanza primaria y secundaria gratuita y obligatoria, de buena calidad con igualdad de géneros y equidad; desarrollando competencias como aprender a aprender que le permitan estar actualizándose a lo largo de su vida. En un aprendizaje continuo a través de tres niveles: "el primero que es el de desarrollo personal y cultural; que le da sentido a la existencia de cada individuo, el segundo; el desarrollo social, que se refiere al lugar ocupado por el individuo dentro de su comunidad y el tercero; del desarrollo profesional que le permite desenvolverse como persona y desempeñar una misión que es útil para el resto de su comunidad". (UNESCO, 2005).

Ahora sería importante analizar si esta sociedad permite la inclusión de todos sus miembros o hay exclusiones; tal como la UNESCO plantea esta Sociedad del Conocimiento con alcances muy altruistas; intenta mediar y exigir a cada país que realmente se imparta una educación que eleve la condición de vida de los ciudadanos. En este mundo en vía de la globalización su intervención es primordial y necesaria sobre todo en los países donde la ambición humana, la corrupción y la violencia han dañado su estructura básica; ahí se requieren retomar el rumbo. La presión y el apoyo de la UNESCO es primordial y de gran utilidad ya que pretende incluir a todos, en el proceso de adquisición de los conocimientos que se requieren, para poder ser competitivos en esta nueva sociedad; aunque esta inclusión deja también un espacio a la exclusión en los lugares donde no se logre dicho objetivo.

Tedesco (2003) también nos habla de la Sociedad del Conocimiento a la que denomina también Nuevo capitalismo en el que se pretende incluir en su proceso solo a los individuos que desarrollen las capacidades básicas vinculadas con la educabilidad y empleabilidad.

Con lo cual se excluye al resto de la población que no tenga las características que este nuevo sistema requiere; marginando o dejando al resto en su mismo nivel de pobreza, ignorancia y violencia.

Binemiles (2010) se refiere más que a una Sociedad del Conocimiento a una economía del conocimiento; que es un nuevo paradigma técnico-económico, donde la ciencia se ha transformado en una de las actividades más productivas; para asegurar el triunfo de este modelo todos coinciden en que es necesario relacionarlo estrechamente con la educación, para que mediante ella se desarrollen las habilidades o competencias necesarias, como la alfabetización digital para preparar personal altamente calificado para la producción; este nuevo sistema está basado en la producción del conocimiento científico, es decir en la acumulación del capital intelectual, para promover la integración funcional productiva, eminentemente con fines empresariales; en el cual únicamente los que desarrollen las competencias requeridas quedan incluidos y serán beneficiados en dicho proceso. 
Otros autores como Andrade y Campo (2008) se refieren a esta nueva sociedad como sociedad de la información. En una investigación que se hace en Venezuela al compararlo con otros países del mundo, coinciden en el aspecto excluyente en cuanto al acceso a la tecnología aplicada a la educación. Debido a que existen diferencias territoriales que limitan el ingreso a los centros educativos en cuanto a su ubicación; los estudiantes que viven en las zonas urbanas se ven privilegiados con las nuevas tecnologías y se excluyen a los alumnos de las zonas rurales y marginales que no tienen acceso a los recursos de infraestructura tecnológica.

Estos autores han utilizado indistintamente diferentes terminologías para designar a esta sociedad predominando la de Sociedad del conocimiento o Sociedad de la información enfocándonos en el significado de dichos conceptos: conocimiento e información consideraríamos que denominar a la sociedad actual Sociedad del Conocimiento sería muy complejo porque abarca un proyecto muy ambicioso, pues hablar del conocimiento en sí es plantearlo como algo acabado, terminado e incambiable; que no es lo que realmente existe, no podemos hablar de una sociedad poseedora de conocimiento, sino que es el ideal en el que se estará en permanente búsqueda y será el generador de la riqueza; analizando solo el significado sería más real hablar de una Sociedad de la información ya que el término “información” es algo aislado que se puede unir, moldear y manipular con el propósito de llegar a construir los conocimientos que se requieran para dar soluciones distintas a problemas diversos. La Sociedad del Conocimiento "se basa en el progreso social, ético y político"; (Vela, 2011).bastante utópico en este mundo tan materializado; mientras que "la sociedad de la información se basa solo en el progreso tecnológico". (Vela, 2001). Lo que realmente existe en la actualidad, es un mundo de información virtual circulando en la red de Internet gracias a la tecnología de la información (TIC), es solo eso, información y el conocimiento que requieren las sociedades del futuro aún esta en permanente construcción.

Aunque el conocimiento ha creado estas nuevas tecnologías, lo que ellas ofrecen es solo información, aunque que en su uso cotidiano y en las relaciones sociales, culturales y económicas han ayudando a eliminar las barreras del espacio y del tiempo, facilitando una mejor y rápida comunicación; modificando con esto la forma en que se desarrollan muchas actividades de la sociedad actual. La tecnología ha cambiado a la sociedad y un grupo selecto de ésta, ha creado dicha tecnología ambas se han influenciado y transformado mutuamente.

\section{Conclusiones}

Existe una preocupación social de organizaciones como la UNESCO por crear una sociedad que brinde las oportunidades educativas ideales, con la finalidad de que el individuo mejore su condiciones de vida, por ello exige a los países atrasados; trabajen en el logro de los avances que les permitan a sus educandos, igualar sus competencias para tener las mismas oportunidades en un mundo globalizado.

Entonces como se debe llamar a esta sociedad que esta realizando cambios para transformarse y prepararse con una visión a futuro, si se analiza solo la semántica para designar a la sociedad actual el término más indicado para calificarla; como Sociedad del Conocimiento o Sociedad de la información, desde la perspectiva de las autoras se considera que el primer término, promete más de lo que ofrece actualmente, porque lo que proporciona las nuevas tecnologías y lo que realmente caracteriza a nuestra sociedad, la gran disponibilidad de información, el término más adecuado si se considera únicamente su significado sería en todo caso, el de Sociedad de la 
Información puesto que es la que navega abundantemente en la redes de Internet y está disponible para todo aquel que tenga los recursos tecnológicos que le permitan accesar a ella; visto de esta manera, se sabe que la Sociedad de la Información aspira a ser una Sociedad del Conocimiento; aunque si se le da preponderancia al factor productivo también es válido el término de Sociedad del Conocimiento por ponderar a este como la fuerza que hegemoniza la reproducción del sistema económico; la innovación da solución a las problemáticas y necesidad del mundo presente y futuro pues actualmente el conocimiento es ya el motor que esta innovando y mejorando la tecnología (Montes, 2001).

Indistintamente de la terminología utilizada es importante concluir si esta sociedad incluye o excluye a todos en los beneficios que pretende ofrecer. $\mathrm{Al}$ analizar el esfuerzo que hace la UNESCO con el apoyo de los gobiernos de sus respectivos países al pretender incluir a todos en equidad y las diversas concepciones de los autores mencionados de lo que es y busca esta sociedad; se observa que toda inclusión deja un margen para la exclusión, al existir restricciones con las que se incluyen a unos y se excluye a otra parte, en este caso se da principalmente por dos motivos: el que algunos individuos no puedan tener acceso a la información, por no contar con los recursos tecnológicos necesarios o bien por no poseer una alfabetización digital, habilidad indispensable que abre las puertas para entrar a ese mundo virtual de la información.

Y aún para quienes cuenten con estas dos factores; no se garantiza que el conocimiento se obtenga, pues hay que saber buscar la información, hilarla y utilizarla para un bien común, con un sentido crítico y un fin utilitario, recurrir a esta para crear saberes; logrando transformarla en conocimiento que sirva para innovar o solucionar una problemática o necesidad, de los ciudadanos del futuro. Entonces es necesario considerar y tener cuidado en el conocimiento pertinente que se debe incluir en los planes y programas del nivel educativo y de la formación docente (Morín, 2000) porque no hay un conocimiento único propuesto solo las características que este debe tener, porque estará en permanente construcción atendiendo necesidades diversas.

Ya que como se ha observado en cada etapa de la humanidad han sido conocimientos diferentes y necesarios los que en su momento, han dado solución a necesidades específicas de la sociedad y es lo que ha permitido el avance de la misma; mejorado sus condiciones de vida, aunque siempre una parte de dicha sociedad ha sido la más beneficiada y es la que ha tomado el liderazgo, es la poseedora y generadora de dichos saberes, que son puestos a disposición de todos como satisfactores. Y aunque en la actualidad sigue existiendo escuelas para una cierta élite que posee los recursos económicos para pagar ese tipo de educación; la que oficialmente proporciona el estado también ofrece múltiples beneficios y a diferencia de antaño ahora esta a disposición de todos los que deseen desarrollar una educación de calidad que le permita entrar al mundo competitivo.

Por esto el compromiso como docentes es mayor, ya que implica preparar hoy, a las nuevas generaciones para satisfacer necesidades que aún no existen; para ello se debe lograr desarrollar en el alumnado todas sus capacidades y competencias que le puedan ayudar a solucionar los retos que se le presenten en su vida futura.

$\mathrm{Y}$ aún que actualmente, algunas escuelas cuentan ya con el innovador recurso didáctico, de las TIC lamentablemente no está a disposición de todos. En México por ejemplo se empezó a dotar de tecnología a las escuelas a partir del año 2003 con el programa Enciclomedia Alaníz (2009). Pero el proyecto concluyó en el 2011, quedando además semi-obsoleto al entrar la nueva reforma educativa y cambiar los libros de texto que estaban digitalizados en este programa. Para solucionar el problema y ampliar su cobertura, se anunció la creación de uno 
nuevo al que se le denominó: Habilidades digitales para todos (HDT), que apoyaría y daría continuidad al anterior, ya que muchos equipos están paralizados por falta de mantenimiento; este programa que pretendía abarcar también al nivel de secundaria, no logró su objetivo en la cobertura anunciada; dotar a las aulas de tecnología es un proceso que avanza muy lentamente, por sus limitantes económicas; pese al esfuerzo de los gobiernos.

En los inicios de este programa y aún hoy, pero en menor grado, se cuenta con otra limitante, pues aún que existe tanta información circulando por la red; pareciera que se está en el medievo donde el conocimiento estaba resguardado en las bibliotecas de los conventos y solo los que poseían el conocimiento de la lectura podían acceder a él, ahora se requiere alfabetización digital, para poder tener disponibilidad al uso didáctico de esta tecnología; habilidades que todas y todos los docentes deberían desarrollar, impulsados por la educación pero con el compromiso y disposición de todos. Para atender esta situación en México se dotó de una laptop a cada maestro (a), para que la falta de tecnología ya no sea una limitante, corresponde ahora a cada uno, en forma individual desarrollar dichas habilidades digitales y buscar su certificación.

Pero aún, imaginando que la tecnología llegara a todos los rincones de la tierra y todos aprendieran a hacer uso de ella, se les estaría proporcionando únicamente información de forma equitativa, con ella se les brindaría las mismas oportunidades para obtener los saberes precisos e indispensables para que cada individuo desarrolle sus habilidades; con las que pueda servir dentro de un campo laboral, al sistema que lo exige y el mundo globalizado requiere, para lograr sobrevivir en esta sociedad competitiva, pero el conocimiento corresponde a cada individuo construirlo e innovarlo.

Desde lo que consideran las autoras se debe reconocer que en la historia de la humanidad se está en el nivel máximo de acercamiento con la información existente, en forma rápida y abundante que podría llegar a ser el libertador del descendiente del antiguo esclavo, siervo y ahora obrero que no necesitará más de la fuerza física para generar mejores posibilidades económicas, solo de su intelecto y el uso que haga de él para generar el conocimiento que satisfaga alguna necesidad social y con ello logrará elevar su nivel de vida.

Por esto los y las docentes de todo el mundo, deben preparase para hacer un mejor uso de las nuevas herramientas tecnológicas y de información, con las que logren alfabetizar digitalmente a los educandos e incluir a todos en el desarrollo de las competencias requeridas, para poder triunfar dentro de esta sociedad (Davini, 2010).

También es necesario implementar nuevas estrategias y promulgar la creación de instituciones o mecanismos, que rescaten al alumnado desertor del sistema escolar, para que no fracasen en el logro de la adquisición del conocimiento y queden todos incluidos en este proceso.

En la nueva Sociedad de la Información, recae en el docente la principal misión de poder en verdad incluir a todos, ya que la educación es la llave para ingresar a esta sociedad, en ello radica la vocación docente en las manos de los y las maestras esta enlazar los nobles propósitos de la UNESCO, con los esfuerzos que realicen los gobiernos para equipar de tecnología las escuelas, ya que con estos nuevos recursos didácticos, se deben transformar al estudiantado que llega a las aulas deseoso de ser guiados en la adquisición de los saberes necesarios, con aprendizajes significativos, para que al aprender haciendo logren desarrollar las habilidades y las competencias requeridas, consiguiendo con ello las herramientas esenciales que les permitirán dar solución y satisfacer las necesidades que surjan en el mundo futuro.

El camino esta marcado a través de la educación, con el apoyo de la tecnología, de los gobiernos y si fuera posible de 
la inversión de las empresas privadas, ya que ellas serán las principales beneficiadas del perfil de egresado que se pretende formar con el esfuerzo en conjunto de todos los involucrados, cuyo objetivo principal debe ser proporcionar educación de calidad en ambientes educativos ideales; será un triunfo de la sociedad y gran satisfacción del magisterio ver aquellos niños y niñas convertidos en ciudadanos respetuosos de su entorno y de la vida, responsables de sus labores, democráticos, críticos, participativos e innovadores con todas las actitudes y saberes adquiridos que les abrirán las puertas con facilidad a su incorporación al sistema productivo en un mundo competitivo y globalizado en el que urge crear las condiciones para poder incluir a todos, para beneficio de toda la sociedad, ya que con ello se lograría; alejar lo más posible a las futuras generaciones de jóvenes de la marginación, la pobreza y la violencia brindándoles realmente oportunidades en una sociedad más equitativa y justa.

\section{Notas}

1 Informe mundial de la UNESCO, 2005. P 29.

2 Informe Mundial de la UNESCO.2005. cap. 4. p 75 y 77.

3 Juan Carlos Tedesco (nacido en 1944) es un educador y pedagogo argentino, que se desempeñó como Ministro de Educación de Argentina.

4 Edgar Morín, director de investigación Emérita, en el Centro Nacional de Investigación Científica y Presidente de la Agencia Europea de la Cultura.

$5 \quad$ Programa que tiene como propósito el uso de la tecnología para enriquecer el proceso educativo: inicia su pilotaje en México durante el ciclo escolar 2003-2004, con la instalación de cerca de 21 mil equipos en las aulas de quinto y sexto grado de primaria.
6 Inicia como profesora de preescolar, alcanza grado de doctorado en educación; obtiene el reconocimiento del Programa de Mejoramiento del Profesorado en Educación Superior. Autora del libro Educación Básica en México. págs. 35, 104-106.

\section{Referencias}

Alaníz, C. (2009). Educación básica en México: De la alternancia al conservadurismo. México, D.F: Gernika.

Andrade, J. A., \& Campo, M. S. (2008). "Tecnología de la información: inclusión en la educación basada en lo digital". Revista Mexicana de Investigación Educativa, 13 (036), 223-248.

Anónimo. (1994-1995). Enciclopedia Hispánica. (32 volúmenes) Kentucky, E.U. Encyclopeadia Britannica Publisher INC.

Binimelis, H. (2010). "Hacia una sociedad del conocimiento como emancipación: una mirada desde la teoría crítica", 023 (62). 203-224. Recuperado de http://redalyc.uaemex.mx/src/inicio/ artpdfred.jsp? ;cve $=59515960009$

Braillard, P., y Senarclens, P. (1982). El imperialismo. México, D.F: Fondo de Cultura Económica.

Contreras, H. (s.f.). "Por qué utilizamos el calendario". La Ciencia y el Hombre. Revista de divulgación Científica y Tecnológica de la Universidad Veracruzana, 018 (1). Recuperado de http://www.uv.mx/cienciahombre/ revistae/vol18num1/articulos/calendario/index.htm

Davini, M. C. (2010). La formación docente en cuestión: política y pedagógica. Buenos Aires Argentina: Paidós.

Garibay, Á. M. (1989). Mitología Griega. Dioses y Héroes. México, D.F: Editorial Porrúa.

Leadbeater, C. (2009). Escuelas secretas de la Masonería. Recuperado de 
http://eruizf.com/masonico/doc/escuela_secreta_de_masoneria_c_w_leadbeater.pdf

Martín, F. (1999). La didáctica ante el tercer milenio. Madrid: Síntesis.

Mautino, J. (2009). Didáctica de la educación tecnológica. 42-48, 58-65 Buenos Aires: Bonum.

Montes, R. (2001). Globalización y nuevas tecnologías: nuevos retos y ¿nuevas reflexiones? Madrid, España: Cuadernos de Iberoamérica, OEI.

Morín, E. (2001). Los siete saberes necesarios para la educación del futuro. México: Dower.

Organización de las Naciones Unidas para la Educación, la Ciencia y la Cultura. (2005). Hacia las sociedades del conocimiento. Informe Mundial. Ediciones UNESCO. Recuperado de http://unesdoc.unesco. org/images/0014/001419/141908s.pdf

Ricardo, D. (2010). Maestros de primaria recibirán en junio laptops ofrecidas por el gobierno federal. Instituto del Libro y la Lectura, A.C. Comentario.
Recuperado de http://www.illac.com. $\mathrm{mx} /$ profiles/blogs/maestros-de-primaria-recibiran

Tedesco, J.C. (2003). "Investigación educativa: de la ciencia social a la filosofía social". Revista Electrónica de Investigación Educativa, 5 (2). Recuperado de http://redie:uabc.mx/ vol5no2/contenido-tedesco.html

Vaillant, G. (1985). La civilización azteca. México, D.F : Fondo de Cultura Económica.

Vargas, J. (s. f.). Neo-Colonialismo, resistencia, crisis y transformaciones del Estado. Colección de documentos, Una publicación del instituto de gobernabilidad de Cataluña. Recuperado de http://www.insumisos.com/lecturasinsumisas/Transformacion $\% 20$ del $\% 20$ estado\%2 0 en $\% 201$ a $\% 20$ crisis\%20neocolonial.pdf

Vela, L. (2011). Sociedad del Conocimiento. Collaboration ideas. Recuperado de http://www.collaborationideas.com/2011/05/sociedad-delconocimiento/?lang $=\mathrm{es}$ 